\title{
Towards Optimisation of Photocurrent from Fullerene Excitons in Organic Solar Cells
}

\author{
Stoichko D. Dimitrov ${ }^{a}$, Zhenggang Huang ${ }^{a}$, Florent Deledalle ${ }^{a}$, Christian B. Nielsen ${ }^{a}$, Bob C. Schroeder \\ ${ }^{a}$, Raja Shahid Ashraf ${ }^{a}$, Safa Shoaee ${ }^{a, b}$, Iain McCulloch ${ }^{a}$ and James R. Durrant ${ }^{a}{ }^{a}$ \\ ${ }_{5}$ Received (in $\left.X X X, X X X\right)$ Xth $X X X X X X X X X 20 X X$, Accepted Xth $X X X X X X X X X 20 X X$ \\ DOI: $10.1039 / \mathbf{b 0 0 0 0 0 0 x}$
}

Photocurrent from fullerene excitons in polymer/fullerene solar cells is optimised by employing a series of low bandgap diketopyrrolopyrrole-containing polymers with differing molecular weights. The low LUMO level of this donor polymer prevents efficient charge generation from polymer excitons, allowing 10 us to focus on charge and photocurrent generation specifically from fullerene excitons. We employ femtosecond transient absorption spectroscopy and transmission electron microscopy to show a correlation between fullerene domain size and the kinetics of polaron generation from fullerene excitons, and relate these observations to device efficiency. Charge generation from fullerene excitons is shown to occur on the $0.5 \mathrm{~ns}$ timescale, limited by the kinetics of diffusion of fullerene excitons to domain

15 interfaces with donor polymers. For devices employing the highest molecular weight donor polymer, $\sim 7$ $\mathrm{mA} . \mathrm{cm}^{-2}$ of photocurrent is observed from fullerene excitons, leading to an overall device efficiency of $5.2 \%$.

\section{Introduction}

Polymer/fullerene organic solar cells (OSC) have the potential to ${ }_{20}$ provide an inexpensive and efficient solar technology. ${ }^{1}$ They consist of a film of a photoactive electron-rich polymer and electron-poor fullerene sandwiched between two electrodes. In the film, both the polymer and fullerene absorb sunlight and their excitons generate charges via a series of charge transfer steps

25 driven by the energy differences of the polymer and fullerene frontier molecular orbitals. ${ }^{2,3}$ Research efforts have in recent years been particularly focused on developing new donor polymers for more efficient light harvesting and charge photogeneration. ${ }^{4-6}$ With the development of these lower bandgap 30 polymers, the role of the fullerene in absorbing higher energy photons has become increasingly important, leading to increasing use of the asymmetric, fullerene, $\mathrm{PC}_{70} \mathrm{BM}$ due to its high absorption coefficient. ${ }^{7}$ However, relatively few studies have focused on investigating and optimising the generation of 35 photocurrent resulting from this fullerene light absorption. ${ }^{8-12}$ Such photocurrent generation can be assigned to dissociation of fullerene excitons at polymer/fullerene interfaces, corresponding to a HOMO to HOMO hole transfer reaction. ${ }^{9,10,13}$

Several studies have considered the importance of fullerene 40 aggregation within the blend film in relation to device performance. ${ }^{12,14-17}$ It has been suggested that fullerene aggregation may be important in stabilising charge separation and providing more efficient charge transport and collection at the electrodes. ${ }^{18}$ Conversely, we and others have identified that 45 fullerene aggregation into domains with diameters bigger than their exciton diffusion length can limit device photocurrents due to inefficient exciton diffusion to the polymer/fullerene interface. ${ }^{8-10,13}$

In this paper, we focus on the kinetics of fullerene exciton ${ }_{50}$ diffusion and charge separation, employing blend films of $\mathrm{PC}_{70} \mathrm{BM}$ with the donor polymer BTT-DPP (see Figure 1 for polymer's structure). We have previously shown that photocurrent generation in OSC employing these photoactive layer materials primarily results from fullerene light absorption, ${ }^{13}$ 55 with BTT-DPP excitons being too low in energy to drive efficient charge separation. ${ }^{19}$ Herein, we employ a series of BTT-DPP fractions with differing molecular weights in order to optimise film microstructure and to investigate the correlation between fullerene singlet exciton dynamics and photocurrent generation. ${ }_{60}$ Following this approach we are able to achieve $\sim 7.3 \mathrm{~mA} \cdot \mathrm{cm}^{-2}$ photocurrents from $\mathrm{PC}_{70} \mathrm{BM}$ excitons alone. We furthermore demonstrate that this impressive photocurrent generation is limited by fullerene exciton dissociation in these devices primarily occurring with a $0.5 \mathrm{~ns}$ time constant. Optimised ${ }_{65}$ devices reached power conversion efficiencies (PCE) of 5.2\%, with device photocurrents resulting primarily from fullerene light absorption.

\section{Results and Discussion}

In the devices employed herein, the primary function of the 70 donor BTT-DPP polymer is as a hole transport material, with the $\mathrm{PC}_{70} \mathrm{BM}$ playing the role of electron transport material and primary light absorber for photocurrent generation. Previously, we have shown that photocurrents from BTT-DPP:PC ${ }_{70} B M$ devices are largely limited by the poor microstructure of the 75 active layer. ${ }^{13}$ To optimise this microstructure, we synthesised BTT-DPP and subsequently fractionated it to yield three BTT- 
DPP fractions with number average molecular weights $\left(M_{n}\right)$ of 22 (BTT-DPP-22), 73 (BTT-DPP-73) and 90 (BTT-DPP-90) kg.mol ${ }^{-}$ ${ }^{1}{ }^{20,21}$ Bulk heterojunction solar cells were fabricated with a standard ITO/PEDOT:PSS/polymer:PC ${ }_{70} \mathrm{BM} / \mathrm{Ca} / \mathrm{Al}$ structure. For 5 all three BTT-DPP fractions, a 1:2 weight ratio (polymer:fullerene) blend composition was found to give optimum device efficiency. Figure 1a shows the $\mathrm{J} / \mathrm{V}$ characteristics of BTT-DPP-22/PC ${ }_{70} \mathrm{BM}$, BTT-DPP-73/PC ${ }_{70} \mathrm{BM}$ and BTT-DPP-90/PC $\mathrm{PC}_{70} \mathrm{BM}$ devices under simulated 1 sun 10 (AM1.5) irradiation, with the corresponding performance data being listed in Table 1. The differences in $\mathrm{J}_{\mathrm{SC}}$ between these devices should be highlighted. The BTT-DPP-90 based device has $\sim 3.5$ times higher photocurrents than the BTT-DPP-22 based device, which can only be partly explained by differences in film 15 thickness (see the supporting information (SI)). However, the open circuit voltage $\left(\mathrm{V}_{\mathrm{OC}}\right)$ and fill factor $(\mathrm{FF})$ of all devices were found to be rather invariant. Therefore, the BTT-DPP-90 based device had a maximum average PCE of $5.2 \%$, whereas the BTTDPP-73 and BTT-DPP-22 based devices had only $2.8 \%$ and $201.5 \%$, respectively. It is also notable that, given these devices were fabricated without any processing additives or annealing steps, their FFs are remarkably high, up to 0.72 , indicating that charge collection in these devices is remarkably efficient, as we investigate further below.
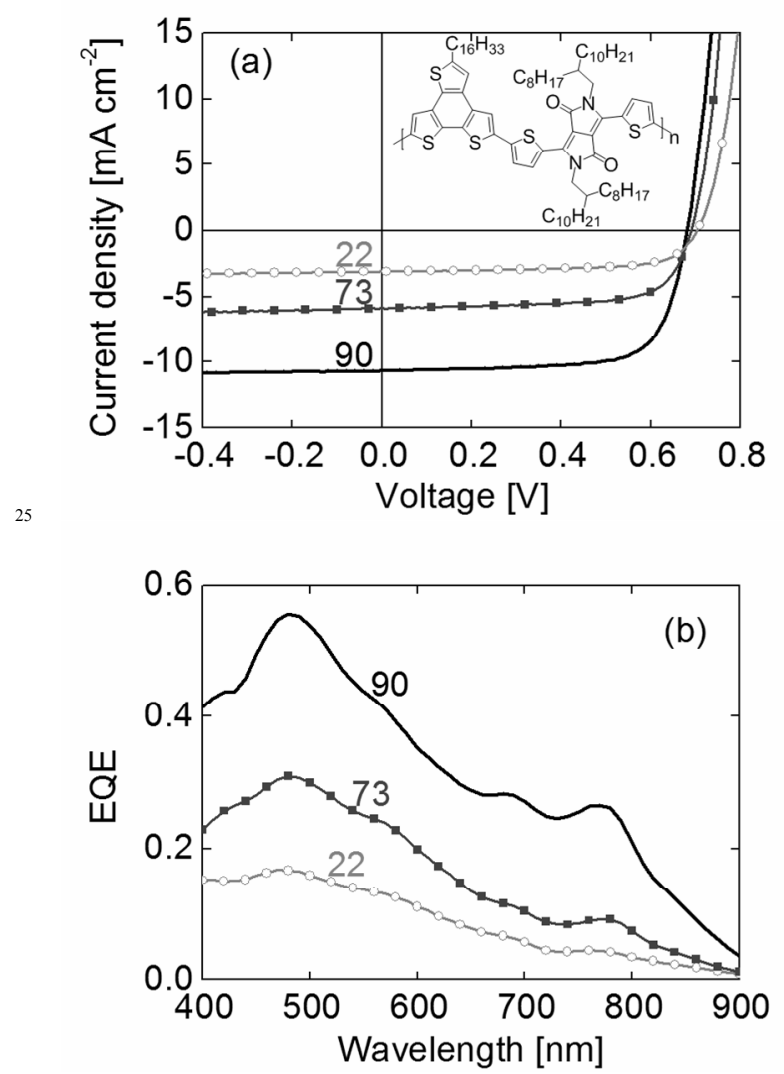

Figure 1. J/V curves (a) and EQE spectra (b) recorded under simulated 1sun AM1.5 illumination for BTT-DPP-22/PC70BM (22), BTT-DPP73/PC70BM (73) and BTT-DPP-90/PC70BM (90) devices (1:2 wt. ratio). 30 For the EQE measurements, cells were kept under constant 1-sun illumination and then exposed to weak monochromatic light.
The external quantum efficiency (EQE) spectra shown in Figure $1 \mathrm{~b}$ primarily track the $\mathrm{PC}_{70} \mathrm{BM}$ absorption spectrum, with 35 only minor contributions from BTT-DPP absorption in the near infrared, as we have discussed previously. ${ }^{13}$ We estimate that by de-convoluting the EQE spectra with the polymer and fullerene absorption spectra (shown in the $\mathrm{SI}$ ) the $\mathrm{PC}_{70} \mathrm{BM}$ light absorption yields on average $70 \pm 15 \%$ of the overall photocurrent generation 40 in the most efficient devices (BTT-DPP-90/PC ${ }_{70} \mathrm{BM}$ ). This leads to photocurrent densities from fullerene excitons alone reaching $\sim 7.3 \mathrm{~mA} . \mathrm{cm}^{-2}$ for the BTT-DPP-90 device.

In order to investigate this trend in photocurrent generation, we carried out femtosecond transient absorption (TA) spectroscopy 45 of thin films of the three different molecular weight blends, as well as neat polymer and fullerene control films. ${ }^{22}$ Blends were excited at $470 \mathrm{~nm}$ (primarily fullerene absorption) and $800 \mathrm{~nm}$ (polymer absorption). The neat fullerene and polymer films were excited at 470 and $800 \mathrm{~nm}$, respectively. In Figure 2a, we 50 compare the TA spectra of the neat BTT-DPP-90 and BTT-DPP$90 / \mathrm{PC}_{70} \mathrm{BM}$ blend films recorded at $0.25 \mathrm{ps}$ and $6.2 \mathrm{~ns}$ delay times, whilst Figures $\mathbf{2 b}$ and $\mathbf{2 c}$ show transient kinetics at selected probe wavelengths.

We consider first the neat film data. No detectable signals $(<$ ${ }_{55} 3 * 10^{-5} \mathrm{OD}$ ) were observed from the neat fullerene film under the relatively low excitation densities employed $\left(7.5 \mu \mathrm{J} . \mathrm{cm}^{-2}\right)$, suggesting that any photoinduced fullerene species exhibit low extinction coefficients relative to polymer excitons/polarons. In contrast, the neat polymer film exhibited a broad positive signal 60 (Figure 2a) assigned to photoinduced absorption of singlet excitons, which was observed to decay mono-exponentially with a 15 ps time constant (Figure 2b). This polymer singlet exciton absorption increases monotonically between 1000 and $1400 \mathrm{~nm}$, and can be readily distinguished from that of BTT-DPP positive ${ }_{65}$ polarons which exhibit an absorption maximum at $1200 \mathrm{~nm} .{ }^{19}$

The blend film showed a more complex TA spectral evolution, as shown in Figure 2a. For both 470 and $800 \mathrm{~nm}$ excitation, the initial $(0.25 \mathrm{ps})$ photoinduced absorption spectrum resembles that of the neat polymer and is therefore assigned to polymer excitons 70 (note that BTT-DPP also absorbs weakly at $470 \mathrm{~nm}$ ). At later times $(6.2 \mathrm{~ns})$ the transient spectra take the shape of BTT-DPP polarons and this is therefore assigned to charge-separated polarons. However, the relative magnitudes of these signals and their temporal evolution depend strongly upon the excitation 75 wavelength (Figures 2a and $\mathbf{2 b}$ ) and polymer molecular weight (Figure 2c).

Figure 2b displays representative transients for the neat BTTDPP-90 film and blend recorded at $1200 \mathrm{~nm}$ for a $470 \mathrm{~nm}$ (fullerene) and $800 \mathrm{~nm}$ (polymer) excitation. It is apparent that, 80 following the $800 \mathrm{~nm}$ excitation, the transient kinetics are dominated by the circa 15 ps decay also observed for neat BTTDPP assigned to polymer singlet exciton decay to ground state, with only a relatively small residual long-lived signal deriving from BTT-DPP polarons. This is consistent with our previous 85 observations of relatively inefficient charge generation from BTT-DPP excitons. ${ }^{13,19}$ In contrast, excitation at $470 \mathrm{~nm}$ results in a pronounced, relatively slow $(\sim 0.5 \mathrm{~ns})$ rise in BTT-DPP polaron signal, indicative of BTT-DPP polaron formation from fullerene singlet excitons. ${ }^{23}$ The large amplitude of this 90 absorption rise (see also Figure 2a) is consistent with our device 
EQE spectra (Figure 1b) indicating relatively efficient charge generation from $\mathrm{PC}_{70} \mathrm{BM}$ excitons, and in agreement with our previous, microsecond timescale, transient studies. ${ }^{13}$ The slow timescale of this rather efficient charge separation $(\sim 0.5 \mathrm{~ns})$ is in 5 marked contrast to the ultrafast (sub-picosecond) charge separation typically observed from most polymer excitons, ${ }^{24,25}$ although examples of slow charge separation from polymer excitons has also been previously observed. ${ }^{26}$ It is striking that charge separation from $\mathrm{PC}_{70} \mathrm{BM}$ excitons is so slow, due to 10 diffusion limitations $(\sim 0.5 \mathrm{~ns})$, but still relatively efficient.
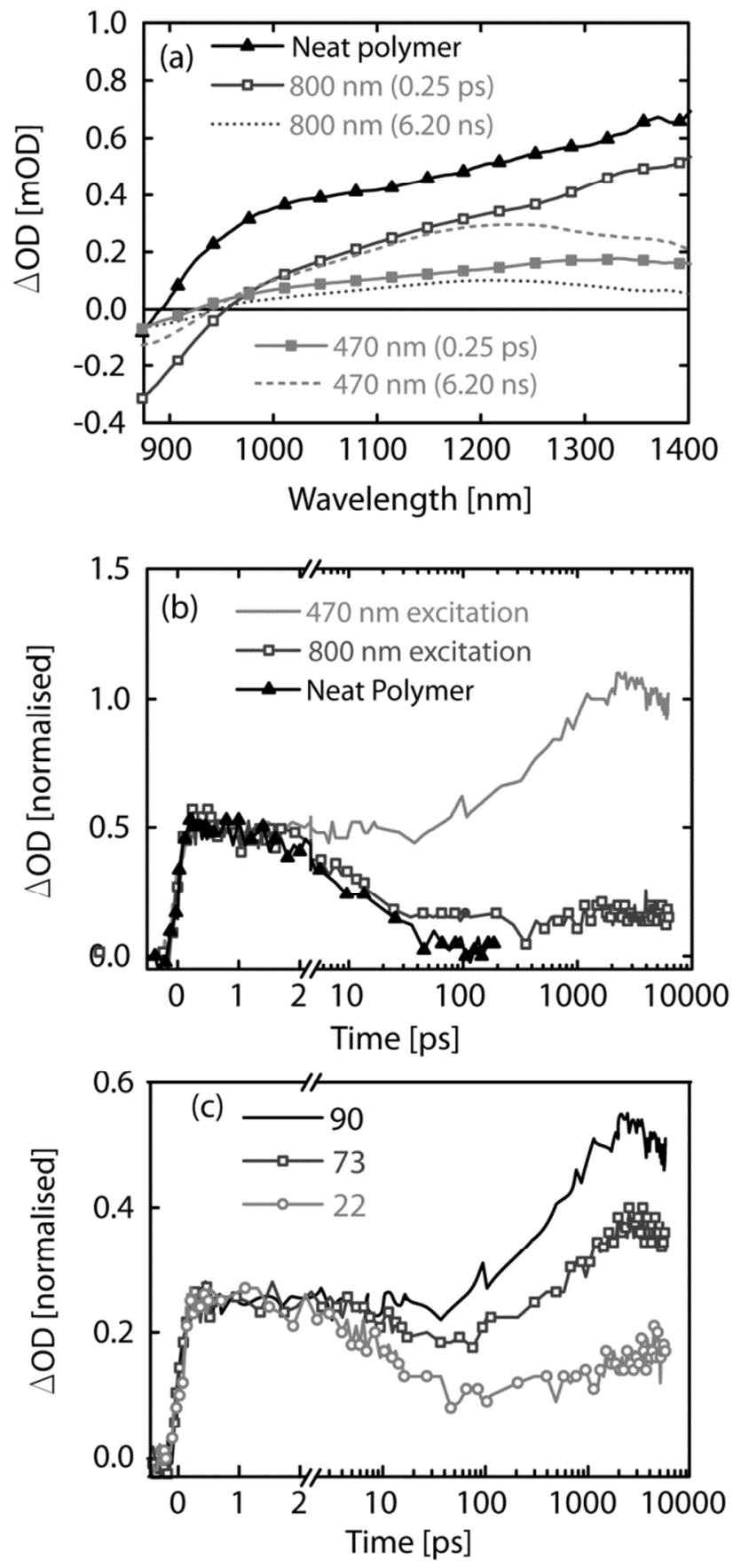

Figure 2. (a) TA spectra of the BTT-DPP-90/PC ${ }_{70} \mathrm{BM}$ blend for a 470 and $800 \mathrm{~nm}$ excitation recorded at $0.2 \mathrm{ps}$ and $6.2 \mathrm{~ns}$ after light absorption. The neat polymer spectrum at $0.25 \mathrm{ps}$ is
15 included as a reference. (b) The corresponding TA kinetics of the BTT-DPP-90/PC ${ }_{70} \mathrm{BM}$ blend and neat polymer measured at 1200 nm. (c) As for (b) but comparing blends with the different $M_{n}$ polymers following $470 \mathrm{~nm}$, excitation. Data in (b) and (c) were normalized at $0.25 \mathrm{ps}$.

20

We now focus on comparing the charge photogeneration properties of the $\mathrm{PC}_{70} \mathrm{BM}$ excitons in the three different molecular weight $\left(\mathrm{M}_{\mathrm{n}}\right) \mathrm{BTT}-\mathrm{DPP} / \mathrm{PC}_{70} \mathrm{BM}$ blends. It is apparent from the absorption rises shown in Figure $2 \mathrm{c}$ that $\mathrm{PC}_{70} \mathrm{BM}$ 25 excitons generate polarons faster and more efficiently in blends with higher molecular weight BTT-DPP. This enhancement of charge photogeneration follows the same trend as the $\mathrm{J}_{\mathrm{SC}}$ in Figure 1a, and is in line with our observation that $\mathrm{PC}_{70} \mathrm{BM}$ excitons are the main contributors to device photocurrents. We

30 note that the only difference between these devices is the molecular weight of the polymer and therefore the most likely cause of this improvement of charge photogeneration is optimisation of the morphology of the films. To verify this hypothesis, we carried out TEM and wide angle X-ray scattering 35 (WAXS) measurements of blend films. TEM micrographs (Figure 3) clearly show a coarser domain structure $(>5 \mathrm{~nm}$ domains) and higher crystallinity for the BTT-DPP-22/PC ${ }_{70} \mathrm{BM}$ film relative to BTT-DPP-90/PC ${ }_{70} \mathrm{BM}$. Similarly, WAXS measurements (Figure S3 in the SI) indicate that the crystallinity 40 in these blends increases in the order $90<73<22$. It is thus apparent that fractionation of BTT-DPP has a significant impact on the morphology of the active layer, decreasing film crystallinity and domain size with increasing polymer $M_{n}$.

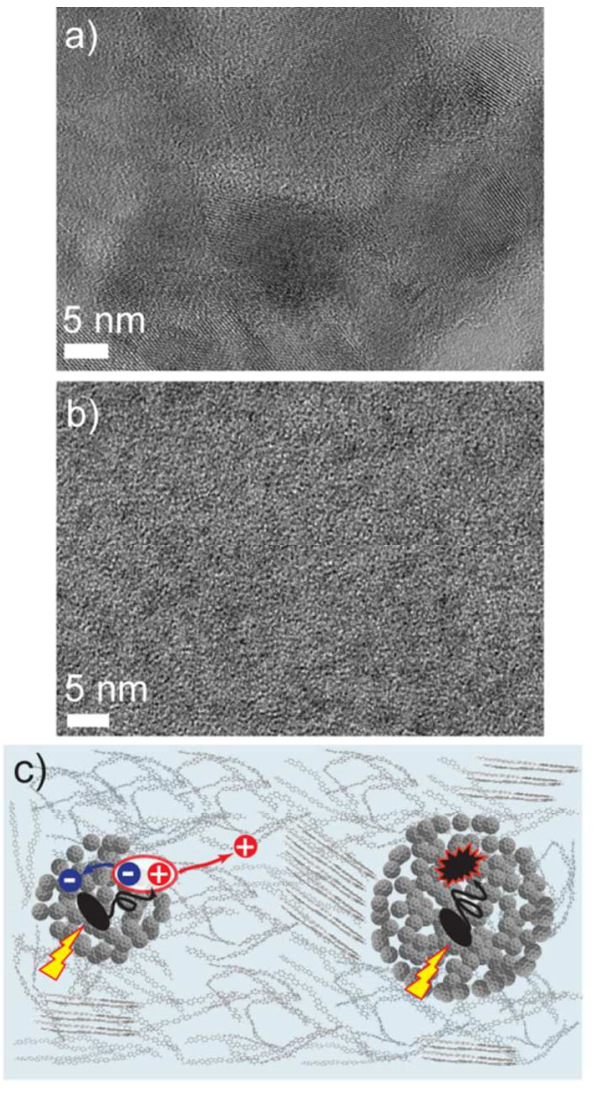

45 Figure 3: TEM images (bright field) of thin films of 1:2 weight ratio BTT-DPP-22/PC 70 BM (a) and BTT-DPP-90/PC ${ }_{70} \mathrm{BM}$ (b) 
blend films. Dark grey patches correspond to $\mathrm{PC}_{70} \mathrm{BM}$-rich domains. (c) Diagram depicting the exciton diffusion process within fullerene aggregates with different diameters. We note that this diagram does not represent realistic film morphology of the 5 studied films, in which the presence of mixed polymer-fullerene phases is likely.

Previous studies have reported fullerene singlet exciton diffusion lengths of $5 \mathrm{~nm}$, of similar order of magnitude to the domain sizes apparent in our TEM images. ${ }^{27}$ In order to 10 investigate this further, we used steady-state photoluminescence (PL) spectroscopy and time-correlated single photon counting to estimate the fullerene singlet excitons and lifetimes in the blends studied herein. The results of these measurements are included in Table 1. They reveal that indeed fullerene singlet excitons are 15 quenched more efficiently and their lifetime is shorter in the more amorphous BTT-DPP/PC $\mathrm{PC}_{70} \mathrm{BM}$ films. We observe a relatively good match between the PL lifetimes and the corresponding charge photogeneration time constants determined from the TA data. Our observed correlation between PL, TEM and TA 20 spectroscopy provides strong evidence that charge photogeneration from fullerene excitons is primarily diffusion limited due to fullerene aggregation on the length scale of the fullerene singlet exciton diffusion length.

\begin{tabular}{|c|c|c|c|c|c|c|c|}
\hline $\begin{array}{c}\mathrm{M}_{\mathrm{n}} \\
\mathrm{kg} / \mathrm{mol}\end{array}$ & $\begin{array}{c}\text { Voc } \\
\mathrm{V}\end{array}$ & $\begin{array}{c}\mathrm{Jsc}_{\mathrm{sc}} \\
\mathrm{mA} / \mathrm{cm}^{2}\end{array}$ & $\begin{array}{c}\mathrm{FF} \\
\%\end{array}$ & $\begin{array}{c}\text { PCE } \\
\%\end{array}$ & $\begin{array}{c}\tau_{\text {rise }} \\
\mathrm{ns}\end{array}$ & $\begin{array}{c}\tau_{\mathrm{PL}} \\
\mathrm{ns}\end{array}$ & PLQ \\
\hline 90 & 0.68 & 10.5 & 0.72 & 5.2 & 0.52 & 0.45 & 0.50 \\
\hline 73 & 0.69 & 5.7 & 0.70 & 2.8 & 0.68 & 0.50 & 0.47 \\
\hline 22 & 0.70 & 2.9 & 0.69 & 1.4 & $\sim$ & 0.53 & 0.32 \\
\hline
\end{tabular}

Table 1. The table presents polymer molecular weights, device $\mathrm{J} / \mathrm{V}$ 25 characteristics, the rise-time ( $\left.\tau_{\text {rise }}\right)$ of the TA (obtained by non-linear least square fitting of the kinetics shown in Figure 2c with two exponential functions convolved with a Gaussian function representing the experimental response function), PCBM photoluminescence lifetime ( $\left.\tau_{\mathrm{PL}}\right)$ and steady state quenching (PLQ). Photoluminescence data is included in

30 the SI. The PL lifetimes ( $\left.\tau_{\mathrm{PL}}\right)$ were calculated from the average of a twoexponential decay fit to the PL decays. $\mathrm{M}_{\mathrm{n}}$ is a number average molecular weight. Error bars of $\tau_{\text {rise }}$ and $\tau_{\mathrm{PL}}: \pm 0.1 \mathrm{~ns}$.

The ability of $\mathrm{PC}_{70} \mathrm{BM}$ singlet excitons to drive reasonably efficient charge separation on relatively long $(0.5 \mathrm{~ns})$ timescales 35 can be understood from its relatively long singlet exciton lifetime. The PL decay of neat $\mathrm{PC}_{70} \mathrm{BM}$ was observed to be $0.65 \mathrm{~ns},{ }^{28}$ indicative of the relatively slow decay of fullerenes singlet excitons compared to many donor polymer excitons (c.f.: 15 ps for the BTT-DPP singlet excitons observed herein). ${ }^{23,29-31}$ The 40 efficiency of fullerene exciton dissociation can be estimated from the steady state PL data to range from $\sim 50 \%$ for the BTT-DPP$90 / \mathrm{PC}_{70} \mathrm{BM}$ blend to $30 \%$ for BTT-DPP-22/PC 70 BM, suggesting that for all the films studied, fullerene exciton decay prior to charge separation remains a significant loss pathway. We note 45 that similar PCBM exciton quenching has been observed for other donor polymers, ${ }^{8,17,23}$ suggesting that our conclusion of significant losses associated with fullerene exciton diffusion limitations may be an important loss factor for many polymer/fullerene solar cells.

50 Optical excitation of neat fullerene films has been reported to result in direct generation of separated charges, albeit with modest quantum efficiencies. ${ }^{32-34}$ Whilst the data reported herein can not rule out low yields of charge generation within PCBM domains, our observation of similar kinetics for both PCBM
55 exciton decay and polymer polaron generation, and similar dependencies upon domain size, indicate that the primary charge generation mechanism in the system studied herein is exciton diffusion to, and charge separation at, the PCBM/polymer interface.
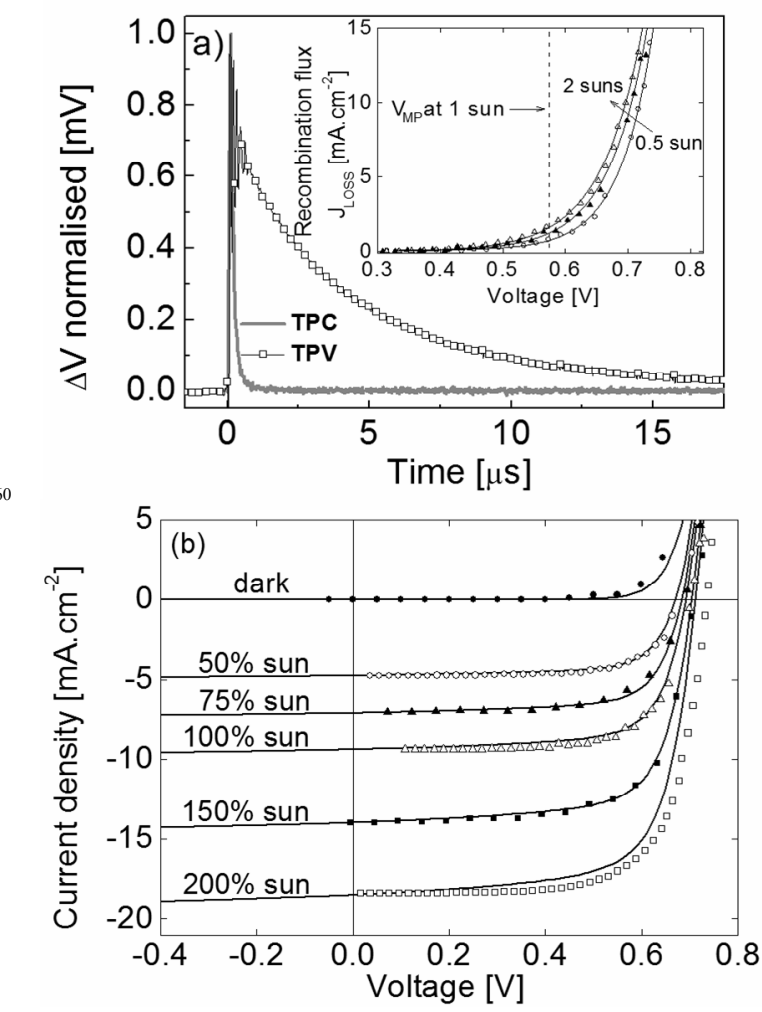

Figure 4. (a) Transient photovoltage and photocurrents. Inset: calculated non-geminate recombination flux $\mathrm{J}_{\text {LOSS }}$ with voltage bias for different light intensities 0.5, 1 and 2 suns (lines are mono-exponential fits). (b) 65 Reconstruction of the current density of BTT-DPP-90/ $/ \mathrm{PC}_{70} \mathrm{BM}$ device in the fourth quadrant in the dark and different light levels ranging from 0.5 to 2 suns. ${ }^{[19]}$ Symbols are experimental data and full lines are reconstructed data.

We turn now to consideration of charge collection in these 70 devices, and particularly our observation of very high FFs and invariable $\mathrm{V}_{\mathrm{OC}}$. To address this, we recorded the $\mathrm{J}_{\mathrm{SC}}$ versus light intensity response of a representative BTT-DPP-90/PC ${ }_{70} \mathrm{BM}$ device from 0 to 4 suns (device $\mathrm{FF}$ and $\mathrm{V}_{\mathrm{OC}}$ did not vary significantly between blends, suggesting that device energetics 75 and charge transport properties were invariant with polymer $M_{n}$ ). The result presented in the SI Figure S4 reveals a remarkably linear response of $\mathrm{J}_{\mathrm{SC}}$ with light intensity $\left(\mathrm{J}_{\mathrm{SC}}\right.$ proportional to $\mathrm{I}^{0.97}$ ) even at 4 suns, indicative of highly efficient charge collection and relatively low non-linear recombination losses at short-circuit in 80 these devices. Furthermore, we used charge extraction and transient photovoltage/photocurrent techniques to quantify charge densities and charge lifetimes in the active layer as a function of voltage and light bias. ${ }^{35}$ Typical photocurrent and photovoltage transients are shown in Figure $4 \mathrm{a}$, showing decay times of $1 * 10^{-7}$ 85 and $5.1 * 10^{-6} \mathrm{~s}$ respectively, with the substantially faster decay time at short circuit being consistent with efficient charge collection. Such transients were employed in the successful reconstruction of the experimental device $\mathrm{J} / \mathrm{V}$ curves (Figure $\mathbf{4 b}$ ) 
and photovoltages (Figure S5 in the SI) in the fourth quadrant for a wide range of illuminations, employing as previously a model that assumes that non-geminate recombination of charges is the only voltage dependent photocurrent loss pathway in the device. ${ }^{36}$

5 The observed excellent match of recreated and experimentally observed $\mathrm{J} / \mathrm{Vs}$, apparent in Figure $4 \mathrm{~b}$, confirms that non-geminate recombination governs the $\mathrm{BTT}-\mathrm{DPP} / \mathrm{PC}_{70} \mathrm{BM}$ device $\mathrm{FF}$ and $\mathrm{V}_{\mathrm{OC}}$. The non-geminate recombination flux (Figure $4 \mathrm{a}$, insert) only becomes significant $(>1 \mathrm{~mA} . \mathrm{cm}-2)$ for voltages greater than ${ }_{10} 0.57 \mathrm{~V}$, approaching $\mathrm{V}_{\mathrm{OC}}$ and consistent with the high device FFs.

The efficient charge collection observed for the devices studied herein, and resultant high device fill factors, can be understood in terms of the kinetics of charge extraction relative to non-geminate recombination. Using charge extraction at short 15 circuit, we estimated that indeed the effective transverse carrier mobility in the BTT-DPP-90/PC $\mathrm{PC}_{70} \mathrm{BM}$ device is $\sim 2 * 10^{-4} \mathrm{~cm}^{2} . \mathrm{V}^{-}$ ${ }^{1} \cdot \mathrm{s}^{-1}$, comparable to $\mathrm{P} 3 \mathrm{HT} .{ }^{37}$ Despite these relatively high charge carrier mobilities, the charge carrier lifetimes are reasonably long, being $\sim 4 * 10^{-6} \mathrm{~s}$ for charge densities of $2 * 10^{16} \mathrm{~cm}^{-3}$ at open-

20 circuit condition, again comparable to P3HT devices, and indicative of a film micro- or interface structure which reduces recombination losses and thereby favors efficient charge collection. $^{38}$

\section{Conclusions}

25 In summary, we have demonstrated the design and characterization of BTT-DPP/PC ${ }_{70} \mathrm{BM}$ photovoltaic devices in which the fullerene excitons alone generate impressive $>7.3$ $\mathrm{mA} . \mathrm{cm}^{-2}$ photocurrents. The low bandgap polymer BTT-DPP was synthesised and fractionated into three different molecular 30 weights to optimise the morphology of the films for more efficient charge photogeneration from $\mathrm{PC}_{70} \mathrm{BM}$ excitons. As a result of this, device $\mathrm{J}_{\mathrm{SC}}$ increased with polymer molecular weight. The most efficient device prepared with the highest molecular weight polymer showed an impressive FF of 0.72 and

${ }_{35} \mathrm{PCE}$ of $5.2 \%$. The high FF results from slow non-geminate recombination losses relative to charge extraction, indicative of a favourable film microstructure for efficient charge collection. Using PL and femtosecond TA spectroscopy, we find that charge generation from $\mathrm{PC}_{70} \mathrm{BM}$ excitons occurs primarily on the $\sim 0.5$ $40 \mathrm{~ns}$ timescale. The higher efficiency, relatively slower, charge generation from fullerene excitons compared to that from BTTDPP excitons is enabled by the slower intrinsic decay time of fullerene singlet excitons ( $0.65 \mathrm{~ns}$ compared to $15 \mathrm{ps}$ for BTTDPP excitons), as well as by the more favourable energetics for ${ }_{45}$ hole rather than electron transfer. The efficiency of $\mathrm{PC}_{70} \mathrm{BM}$ exciton driven charge separation is observed to be dependent upon polymer molecular weight, assigned to variations in the $\mathrm{PC}_{70} \mathrm{BM}$ domain size on the length scale of the $\mathrm{PC}_{70} \mathrm{BM}$ singlet exciton diffusion length. Our results demonstrate that efficient 50 photocurrent generation in polymer/fullerene OSCs can be achieved even with relatively slow exciton-diffusion-limited charge separation kinetics. They also emphasize that light absorption by fullerenes can result in high photocurrents, with the efficiency of this process being dependent in particular upon the 55 efficiency of fullerene exciton diffusion to the charge separation interface, and therefore dependent upon fullerene domain size.

\section{Experimental}

\section{Device preparation}

60 Optimized devices were fabricated with the fractionated BTTDPP polymers co-dissolved with $\mathrm{PC}_{70} \mathrm{BM}$ (Solenne $\mathrm{BV}$ ) in a chloroform/o-dichlorobenzene (4:1) mixed solvent with a weight ratio of $1: 2$ and a $25 \mathrm{mg} / \mathrm{ml}$ concentration. ITO substrates with sheet resistance $15 \Omega \mathrm{sq}^{-1}$ (PsioTec Ltd, UK) were sonicated in ${ }_{65}$ detergent, acetone and isopropanol sequentially and then treated in an oxygen plasma asher (100 W, $7 \mathrm{~min})$. PEDOT:PSS was spin-coated over the ITO substrates at $3500 \mathrm{rpm}$ and baked on a hot plate at $150^{\circ} \mathrm{C}$ in air for $20 \mathrm{~min}$. The BTT-DPP/PC $70 \mathrm{BM}$ solutions were spin-coated onto the PEDOT:PSS layer at the 70 optimum for this blend $3000 \mathrm{rpm}$ in air and transferred to a nitrogen glove box $\left(<10 \mathrm{ppm} \mathrm{O}_{2}, \mathrm{H}_{2} \mathrm{O}\right)$, where $\mathrm{Ca} / \mathrm{Al}(25 \mathrm{~nm} / 100$ $\mathrm{nm}$ ) electrodes were deposited by thermal evaporation under vacuum. Device area was $0.045 \mathrm{~cm}^{2}$.

\section{Device and film characterisation}

$75 \mathrm{~J} / \mathrm{V}$ curves were measured with a $150 \mathrm{~W}$ xenon arc lamp filtered to simulate AM1.5 conditions (LOT Oriel) and a Keithley 238 source measure unit. EQE spectra were measured using a 100 W Tungsten-Halogen lamp. The photocurrent was measured using a Keithley 2400 source meter. A calibrated silicon 80 photodiode with appropriate long pass filters was used as a reference in the $\mathrm{J} / \mathrm{V}$ and $\mathrm{EQE}$ measurements.

PL spectra were measured with a Fluorolog-3 spectrofluorometer (Horiba Jobin Yvon) with a $470 \mathrm{~nm}$ monochromatic excitation. PL dynamics were recorded using ${ }_{85}$ Time-Correlated Single Photon Counting, employing a Horiba Jobin Yvon IBH Fluorocube system. Excitation was 467 nm, 1 $\mathrm{MHz}$ repetition rate and $80 \mu \mathrm{W} . \mathrm{cm}^{-2}$. Decays were recorded at $700 \mathrm{~nm}$, the maximum $\mathrm{PC}_{70} \mathrm{BM}$ PL.

\section{Femtosecond transient absorption spectroscopy}

90 Thin films of BTT-DPP-22/PB ${ }_{70} \mathrm{BM}$, BTT-DPP-73/PB ${ }_{70} \mathrm{BM}$ and BTT-DPP-90/PB ${ }_{70} \mathrm{BM}$ blends were prepared on a glass substrate following the same procedure used during device fabrication. Samples were excited with $470 \mathrm{~nm}$ and $800 \mathrm{~nm} \sim 7.5$ $\mu \mathrm{J} . \mathrm{cm}^{-2}$ pulses, generated by a commercially available optical ${ }_{95}$ parametric amplifier TOPAS (Light conversion) pumped by a Solstice Ti:Sapphire regenerative amplifier (Newport Ltd). Changes in the optical density of the films induced by the laser excitation were followed with a second broadband pulse (830$1450 \mathrm{~nm}$ ) generated in a sapphire crystal. HELIOS (Ultrafast 100 systems) transient absorption spectrometer was used for recording the dynamics of the transient absorption spectra up to $6.5 \mathrm{~ns}$ with average $200 \mathrm{fs}$ IRF.

\section{Wide angle X-ray scattering (WAXS)}

Samples for WAXS measurements were prepared in a drop105 casting manner with the solutions used for device fabrication. The measurements were carried out with a Panalytical X'Pert-Pro MRD which has a nickel-filter $\mathrm{Cu} \mathrm{K} \alpha 1$ beam and an X'Celerator detector, using a current of $40 \mathrm{~mA}$ and an accelerating voltage of $40 \mathrm{kV}$.

\section{Transmission electron microscopy}

For TEM examination, BTT-DPP solutions were spun onto 
PEDOT:PSS-coated $(10 \mathrm{~nm})$ glass substrates, retrieved on holey carbon films. The sample film thickness was $\sim 30 \mathrm{~nm}$. The samples were then stained by $\mathrm{RuO}_{4}$ vapour to increase the contrast of fullerene and polymer domains and measured with 5 Titan 80/300 transmission electron microscope.

\section{Acknowledgements}

We thank the EPSRC APEX project EP/H040218/1, the National Research Fund of Luxembourg and Solvay SA for funding. We also thank Pabitra Tuladhar for device fabrication and Daniel 10 Beatrup for CV measurements.

\section{Notes and references}

${ }^{a}$ Centre for Plastic Electronics, Department of Chemistry, Imperial College London, Exhibition Road, London SW7 2AZ, United Kingdom E-mail:j.durrant@imperial.ac.uk

$15{ }^{b}$ National Physical Laboratory (NPL), Teddington, United Kingdom

$\uparrow$ Electronic Supplementary Information (ESI) available: ESI includes details of polymer GPC traces, molecular weights and PDI, UV/Vis absorbance spectra of blend films, photoluminescence spectra and decays, Wide angle X-ray scattering data, transient photovotlage and charge 20 extraction analysis. See DOI: 10.1039/b000000x/

1. B. Azzopardi, C. J. M. Emmott, A. Urbina, F. C. Krebs, J. Mutale and J. Nelson, Energy \& Environmental Science, 2011, 4, 3741-3753.

25 2. J.-L. Bredas, J. E. Norton, J. Cornil and V. Coropceanu, Accounts of Chemical Research, 2009, 42, 1691-1699.

3. T. M. Clarke and J. R. Durrant, Chemical Reviews, 2010, 110, 67366767.

4. H. Bronstein, Z. Y. Chen, R. S. Ashraf, W. M. Zhang, J. P. Du, J. R. 30 Durrant, P. S. Tuladhar, K. Song, S. E. Watkins, Y. Geerts, M. M. Wienk, R. A. J. Janssen, T. Anthopoulos, H. Sirringhaus, M. Heeney and I. McCulloch, Journal of the American Chemical Society, 2011, 133, 3272-3275.

5. R. Kroon, M. Lenes, J. C. Hummelen, P. W. M. Blom and B. De Boer, Polymer Review, 2008, 48, 531-582.

6. T. Y. Chu, J. P. Lu, S. Beaupre, Y. G. Zhang, J. R. Pouliot, S. Wakim, J. Y. Zhou, M. Leclerc, Z. Li, J. F. Ding and Y. Tao, Journal of the American Chemical Society, 2011, 133, 4250-4253.

7. M. M. Wienk, J. M. Kroon, W. J. H. Verhees, J. Knol, J. C.

40 Hummelen, P. A. van Hal and R. A. J. Janssen, Angewandte ChemieInternational Edition, 2003, 42, 3371-3375.

8. G. F. Burkhard, E. T. Hoke, S. R. Scully and M. D. McGehee, Nano Letters, 2009, 9, 4037-4041.

9. A. A. Bakulin, J. C. Hummelen, M. S. Pshenichnikov and P. H. M.

45 van Loosdrecht, Advanced Functional Materials, 2010, 20, 16531660 .

10. S. Cook, R. Katoh and A. Furube, Journal of Physical Chemistry C, 2009, 113, 2547-2552.

11. T. J. K. Brenner, Z. Li and C. R. McNeill, Journal of Physical Chemistry C, 2011, 115, 22075-22083.

12. B. A. Collins, Z. Li, J. R. Tumbleston, E. Gann, C. R. McNeill and H. Ade, Advanced Energy Materials, 2013, 3, 65-74.

13. S. D. Dimitrov, C. B. Nielsen, S. Shoaee, P. S. Tuladhar, J. Du, I. McCulloch and D. J. R., Journal of Physical Chemistry Letters, 2012, $3,140-144$.

14. F. Piersimoni, S. Chambon, K. Vandewal, R. Mens, T. Boonen, A. Gadisa, M. Izquierdo, S. Filippone, B. Ruttens, J. D'Haen, N. Martin, L. Lutsen, D. Vanderzande, P. Adriaensens and J. V. Manca, Journal of Physical Chemistry C, 2011, 115, 10873-10880.

60 15. T. J. Savenije, W. J. Grzegorczyk, M. Heeney, S. Tierney, I. McCulloch and L. D. A. Siebbeles, Journal of Physical Chemistry C, 2010, 114, 15116-15120.

16. W. L. Rance, A. J. Ferguson, T. McCarthy-Ward, M. Heeney, D. S. Ginley, D. C. Olson, G. Rumbles and N. Kopidakis, ACS Nano,

65 2011, 5, 5635-5646.
17. J. K. J. van Duren, X. N. Yang, J. Loos, C. W. T. Bulle-Lieuwma, A. B. Sieval, J. C. Hummelen and R. A. J. Janssen, Advanced Functional Materials, 2004, 14, 425-434.

18. F. C. Jamieson, E. B. Domingo, T. McCarthy-Ward, M. Heeney, N. Stingelin and J. R. Durrant, Chemical Science, 2012, 3, 485-492.

19. S. D. Dimitrov, A. A. Bakulin, C. B. Nielsen, B. C. Schroeder, J. P. Du, H. Bronstein, I. McCulloch, R. H. Friend and J. R. Durrant, Journal of the American Chemical Society, 2012, 134, 18189-18192.

20. C. J. Brabec, M. Heeney, I. McCulloch and J. Nelson, Chemical Society Reviews, 2011, 40, 1185-1199.

21. R. S. Ashraf, B. C. Schroeder, H. A. Bronstein, Z. Huang, S. Thomas, R. J. Kline, C. J. Brabec, P. Rannou, T. D. Anthopoulos, J. R. Durrant and I. McCulloch, Advanced Materials, 2013, 25, 20292034.

80 22. H. Ohkita and S. Ito, Polymer, 2011, 52, 4397-4417.

23. F. Etzold, I. A. Howard, N. Forler, D. M. Cho, M. Meister, H. Mangold, J. Shu, M. R. Hansen, K. Muellen and F. Laquai, Journal of the American Chemical Society, 2012, 134, 10569-10583.

24. F. Etzold, I. A. Howard, R. Mauer, M. Meister, T. D. Kim, K. S. Lee,

85 N. S. Baek and F. Laquai, Journal of the American Chemical Society, 2011, 133, 9469-9479.

25. M. Tong, N. E. Coates, D. Moses, A. J. Heeger, S. Beaupre and M. Leclerc, Physical Review B, 2010, 81, 125210.

26. C. R. McNeill, S. Westenhoff, C. Groves, R. H. Friend and

90 N. C. Greenham, Journal of Physical Chemistry C, 2007, 111, 19153-19160.

27. S. Cook, A. Furube, R. Katoh and L. Y. Han, Chemical Physics Letters, 2009, 478, 33-36.

28. A. Fedorov, M. N. BerberanSantos, J. P. Lefevre and B. Valeur, 95 Chemical Physics Letters, 1997, 267, 467-471.

29. D. Jarzab, F. Cordella, J. Gao, M. Scharber, H. J. Egelhaaf and M. A. Loi, Advanced Energy Materials, 2011, 1, 604-609.

30. A. J. Lewis, A. Ruseckas, O. P. M. Gaudin, G. R. Webster, P. L. Burn and I. D. W. Samuel, Organic Electronics, 2006, 7, 452-456.

100 31. A. L. Ayzner, S. C. Doan, B. T. de Villers and B. J. Schwartz, Journal of Physical Chemistry Letters, 2012, 3, 2281-2287.

32. S. Kazaoui, N. Minami, Y. Tanabe, H. J. Byrne, A. Eilmes and P. Petelenz, Physical Review B, 1998, 58, 7689-7700.

33. G. F. Burkhard, E. T. Hoke, Z. M. Beiley and M. D. McGehee Journal of Physical Chemistry C, 2012, 116, 26674-26678.

34. W. I. Jeong, Y. E. Lee, H. S. Shim, T. M. Kim, S. Y. Kim and J. J. Kim, Advanced Functional Materials, 2012, 22, 3089-3094.

35. C. G. Shuttle, A. Maurano, R. Hamilton, B. O'Regan, J. C. de Mello and J. R. Durrant, Applied Physics Letters, 2008, 93, 183501

110 36. D. Credgington and J. R. Durrant, Journal of Physical Chemistry Letters, 2012, 3, 1465-1478.

37. C. G. Shuttle, R. Hamilton, J. Nelson, B. C. O'Regan and J. R. Durrant, Advanced Functional Materials, 2010, 20, 698-702.

38. S. A. Hawks, F. Deledalle, J. Yao, D. G. Rebois, G. Li, J. Nelson, Y. Yang, T. Kirchartz and J. R. Durrant, Advanced Energy Materials, 2013, 3, 1201-1209. 


\section{Towards Optimisation of Photocurrent from Fullerene Excitons in Organic Solar Cells}

Stoichko D. Dimitrov ${ }^{a}$, Zhenggang Huang ${ }^{a}$, Florent Deledalle ${ }^{a}$, Christian B. Nielsen ${ }^{a}$, Bob C. Schroeder ${ }^{a}$, Raja Shahid Ashraf ${ }^{a}$, Safa Shoaee ${ }^{a, b}$, Iain McCulloch ${ }^{a}$ and James R. Durrant ${ }^{* a}$

5

Supporting information

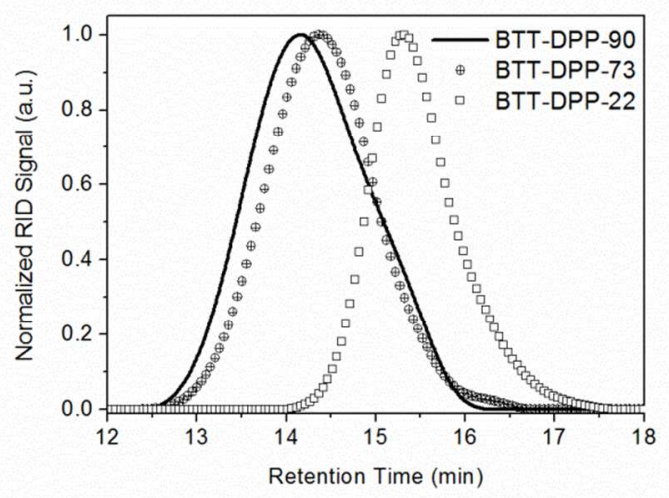

${ }_{10}$ Figure S1. GPC traces for the BTT-DPP fractions with number average molecular weights 22 (BTTDPP-22), 73 (BTT-DPP-73) and 90 (BTT-DPP-90). 

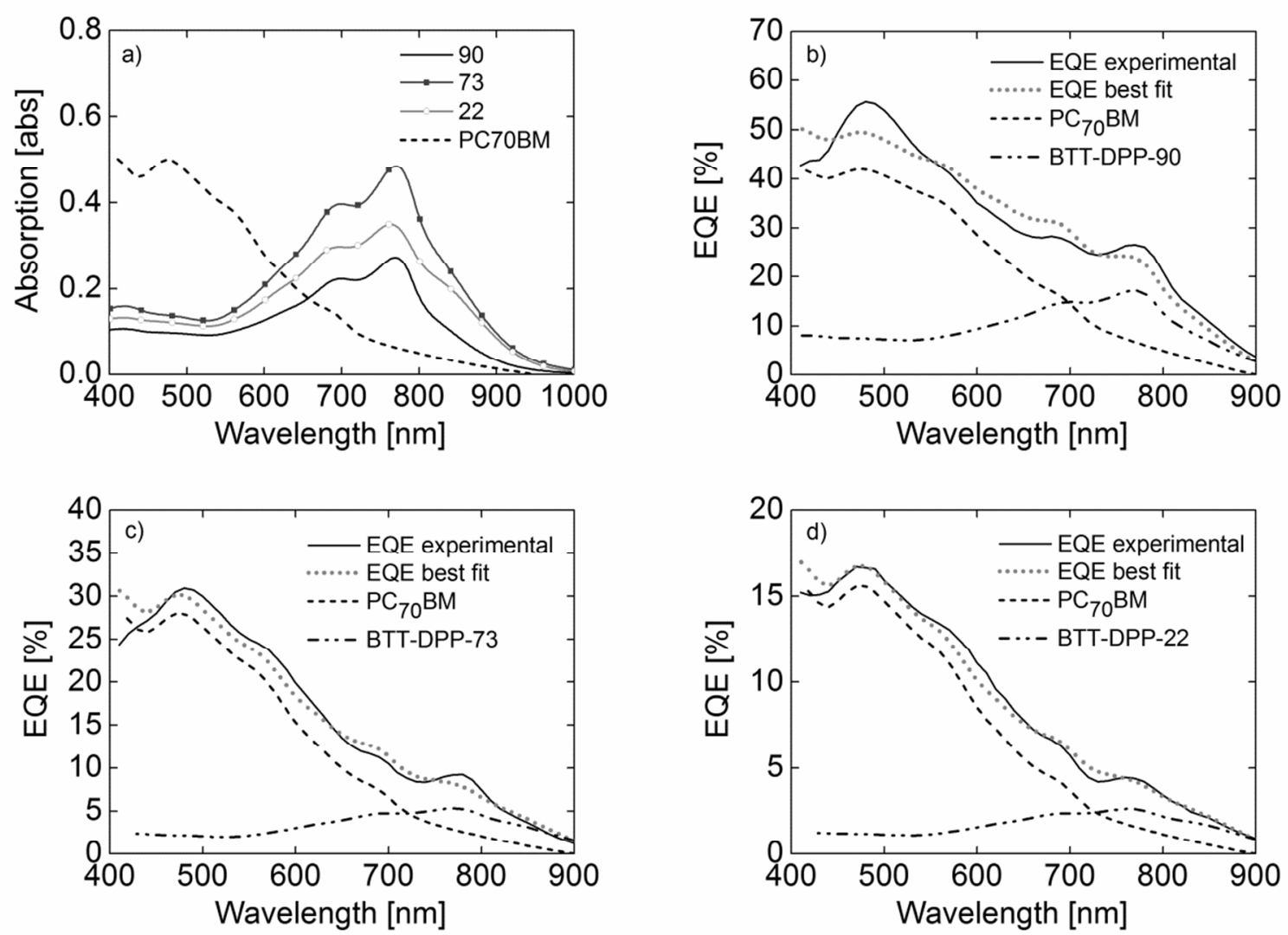

Figure S2: a) Steady-state absorption spectra of thin films of the neat BTT-DPP-90, BTT-DPP-73, BTT-DPP-22 and PC ${ }_{70}$ BM films. Experimentally determined EQE spectra of BTT-DPP-90/PC ${ }_{70} B M$, ${ }_{5} \mathrm{BTT}-\mathrm{DPP}-73 / \mathrm{PC}_{70} \mathrm{BM}$ and BTT-DPP-22/PC $70 \mathrm{BM}$ devices are plotted in $\left.\mathrm{b}\right), \mathrm{c}$ ) and d), respectively. Non-linear least square fits of the EQE spectra and de-convoluted spectral contributions of $\mathrm{PC}_{70} \mathrm{BM}$ and the polymers are also included in the graphs. The spectral contributions of $\mathrm{PC}_{70} \mathrm{BM}$ and BTTDPP-polymers were received by non-linear least-square fitting of their corresponding EQE spectra with the steady-state absorption spectra of the polymer and fullerene shown on Figure S2a. Error bars ${ }_{10}$ of the fits were $15 \%(b), 10 \%(c), 10 \%(d)$. We note that the absorption spectra used in these fits were measured on films and the corresponding fits do not take into account interference effects due to the presence of electrodes. 


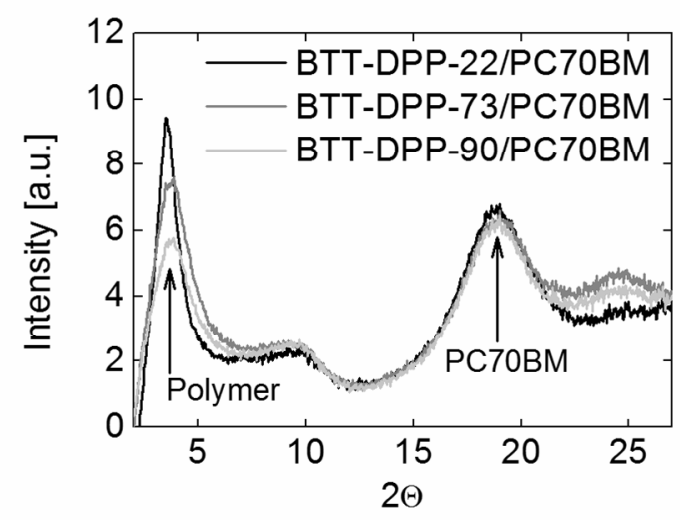

Figure S3: Wide angle X-ray scattering patterns of drop-casted films of fractionated BTT$\mathrm{DPP} / \mathrm{PC}_{70} \mathrm{BM}$ blend films on $\mathrm{Si} / \mathrm{SiO}_{2}$ substrates. Polymer lamellar and $\mathrm{PC}_{70} \mathrm{BM}$ stacking peaks are s marked with arrows. The increase in amplitude and decrease in FWHM of these peaks show that order in the films is increasing.

\begin{tabular}{|l|l|l|}
\hline $\begin{array}{l}\mathrm{M}_{\mathrm{n}} \\
{\left[\mathrm{kg} \cdot \mathrm{mol}^{-1}\right]}\end{array}$ & $\begin{array}{l}\mathrm{M}_{\mathrm{W}} \\
{\left[\mathrm{kg} \cdot \mathrm{mol}^{-1}\right]}\end{array}$ & $\begin{array}{l}\text { Film thick. } \\
{[\mathrm{nm}]}\end{array}$ \\
\hline 90 & 188 & 100 \\
\hline 73 & 155 & 80 \\
\hline 22 & 34 & 50 \\
\hline
\end{tabular}

Table S1. The table presents polymer molecular weights and thicknesses of the films as measured with a Veeco Dektak profilometer. $M_{W}$ is the weight average molecular weight of the polymer. $M_{n}$ is the ${ }_{10}$ number average molecular weight. Our previous estimates of device $\mathrm{J}_{\mathrm{SC}}$ for many different systems (using the matrix-modelling technique under the assumption of $100 \% \mathrm{IQE}$ ) as a function of thickness show that the observed here almost 4-fold increase in $\mathrm{J}_{\mathrm{SC}}$ from BTT-DPP-22/PC 70 BM to BTT-DPP90/ $\mathrm{PC}_{70} \mathrm{BM}$ device (see Figure 1 and Table 1 in the main text) have origin other than changes in device thickness as confirmed by our TAS analysis. 


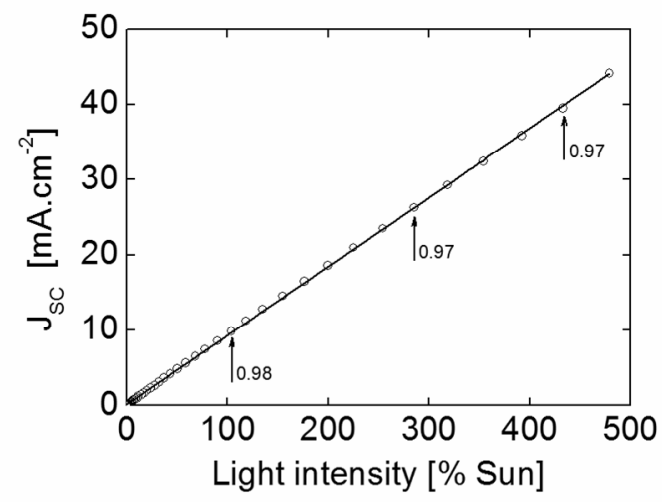

Figure S4: BTT-DPP-90/PC ${ }_{70} \mathrm{BM}$ device $\mathrm{J}_{\mathrm{SC}}$ vs. light intensity. The arrows point out the result of power law fits over different range of illuminations.

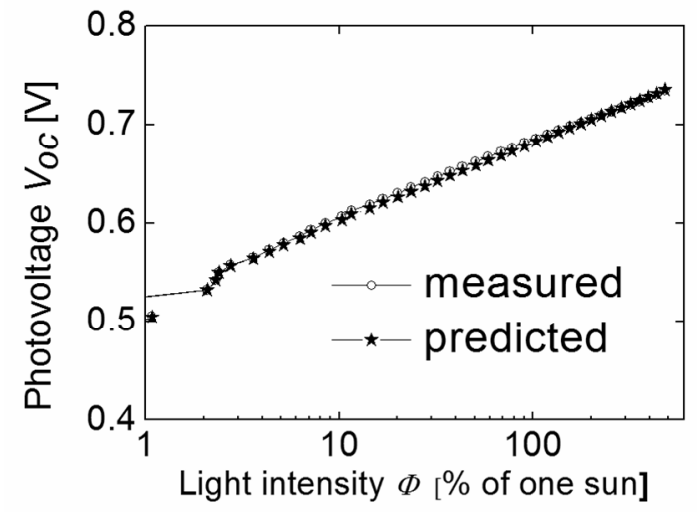

Figure S5: Prediction of the photovoltage based on measurements of charge density at open-circuit and carrier lifetime for the illumination range $1 \%-600 \%$ of one sun for the BTT-DPP-90/PC 70 BM device.

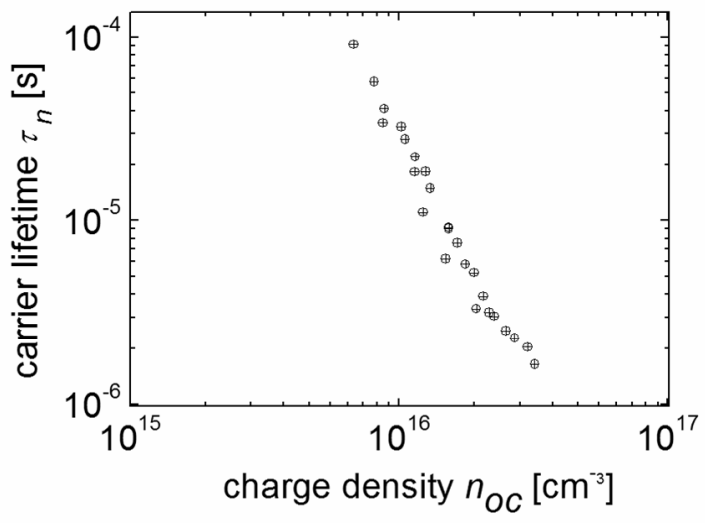


Figure S6: Lifetime of total charge carriers at different charge densities measured using charge extraction at open-circuit and transient photovoltage for the BTT-DPP-90:PC $\mathrm{P}_{70} \mathrm{BM}$ device.
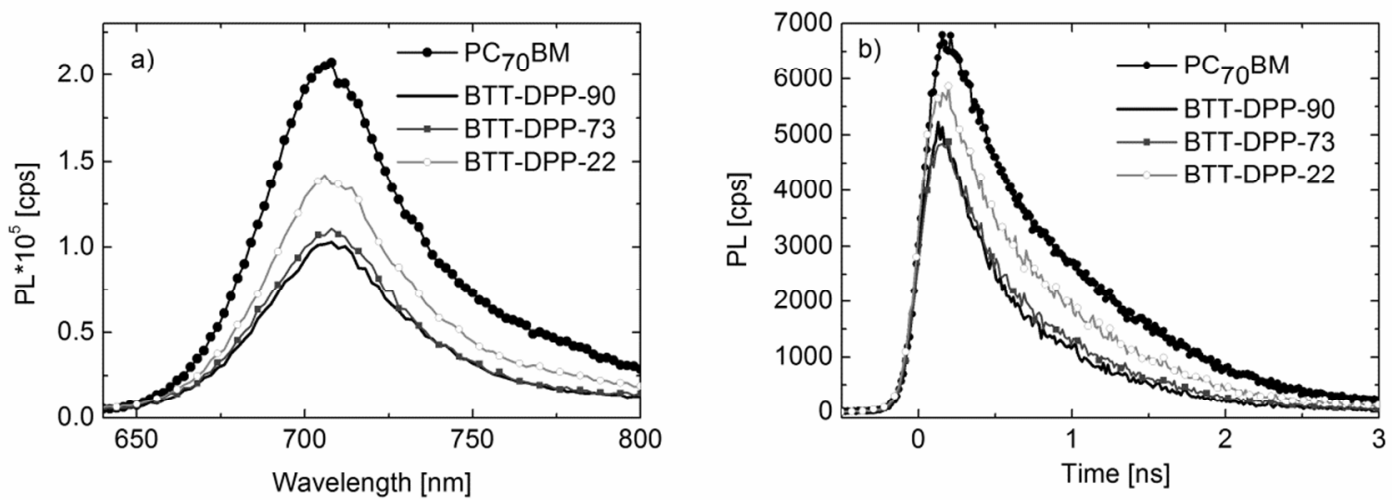

Figure S7: Photoluminescence spectra (a) and decays (b) of BTT-DPP-90:PC ${ }_{70} \mathrm{BM}$, BTT-DPPs. 73: $\mathrm{PC}_{70} \mathrm{BM}, \mathrm{BTT}-\mathrm{DPP}-22: \mathrm{PC}_{70} \mathrm{BM}$ blends and neat $\mathrm{PC}_{70} \mathrm{BM}$ films. Decays were acquired using TCSPC technique with a $200 \mathrm{ps}$ instrument response function. The excitation wavelengths for both experiments were 470 and $467 \mathrm{~nm}$. 
Cite this: DOI: $10.1039 / \mathrm{c0xx} 00000 x$

www.rsc.org/xxxxxx

ARTICLE TYPE

Towards Optimisation of Photocurrent from Fullerene Excitons in Organic Solar Cells

Stoichko D. Dimitrov ${ }^{a}$, Zhenggang Huang ${ }^{a}$, Florent Deledalle ${ }^{a}$, Christian B. Nielsen ${ }^{a}$, Bob C. Schroeder ${ }^{a}$, Raja Shahid Ashraf ${ }^{a}$, Safa Shoaee ${ }^{a, b}$, Iain McCulloch ${ }^{a}$ and James R. Durrant ${ }^{* a}$

TOC

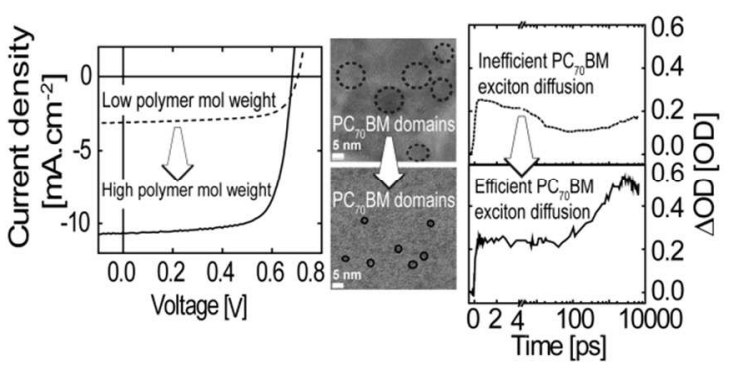




\title{
Towards Optimisation of Photocurrent from Fullerene Excitons in Organic Solar Cells
}

\author{
Stoichko D. Dimitrov ${ }^{a}$, Zhenggang Huang ${ }^{a}$, Florent Deledalle ${ }^{a}$, Christian B. Nielsen ${ }^{a}$, Bob C. Schroeder \\ ${ }^{a}$, Raja Shahid Ashraf ${ }^{a}$, Safa Shoaee ${ }^{a, b}$, Iain McCulloch ${ }^{a}$ and James R. Durrant* ${ }^{* a}$
}

\section{Broader context paragraph}

The sun is our most sustainable energy source and can be effectively used for an off-grid and on-grid ${ }_{10}$ power supply. However, current solar cell technologies still do not provide low cost power to be able to compete with fossil fuels. A particularly popular approach to reducing the cost of solar cells, gaining a high momentum in the past 10 years, is the use of organic materials as the photoactive component. Such organic solar cells can be fabricated to be light-weight, thin and flexible, thus making them ideal for off-grid applications and building integration. Polymer/fullerene solar cells are a type of organic ${ }_{15}$ Solar cells that are now reaching $10 \%$ power conversion efficiency. In these solar cells, polymers and fullerenes absorb sunlight and generate photocurrent, but the way they do that and what limits their photocurrent generation properties is still currently widely debated and as a result difficult to control. In this paper, we explore the limitations of photocurrent generation from fullerene excitons. We apply time-resolved laser spectroscopy and opto-electronic experimental techniques to show that fullerene ${ }_{20}$ aggregation is the main cause for reduced photocurrent generation from fullerene excitons. We show that by careful control over polymer molecular weight, the photocurrent from fullerene excitons can be optimised to give solar cell devices reaching $5 \%$ efficiency. 